\title{
Religiosität und Spiritualität in der interkulturellen Psychotherapie
}

\section{Wirkungen, Methoden und die Identität des/der Therapeut*in}

\author{
Wielant Machleidt \\ Psychotherapie-Wissenschaft 9 (1) 15-21 2019 \\ www.psychotherapie-wissenschaft.info \\ CC BY-NC-ND \\ https://doi.org/10.30820/1664-9583-2019-1-15
}

\begin{abstract}
Zusammenfassung: Die gesellschaftliche Interkulturalisierung und der grössere Einfluss des Islam und anderen Glaubensrichtungen im öffentlichen und privaten Raum in Mitteleuropa haben den religiösen Orientierungen auch von Psychotherapeut*innen und ihren interkulturellen Patient*innen eine grössere Aktualität gegeben. In empirischen Studien konnte nachgewiesen werden, dass ausgeprägte Glaubensüberzeugungen religionsübergreifend für depressive, Angst- und Somatisierungsstörungen sowie Sucht mit einer erhöhten psychischen Resilienz korrelieren. Religionen lassen sich als plurale Symbolsysteme verstehen, die eine Weltordnung mit einem Lebensstil verbinden. Die subtile Kenntnis der Glaubensgewissheiten erlaubt einen Blick auf die innerpsychischen Abläufe und eröffnet breite und individuelle Spielräume für therapeutische Interventionen unter Einbeziehung von Religiosität und Spiritualität. Für die positive Beziehungsknüpfung zum/r Patienten/in ist die Bewältigung der interreligiösen Fremdheitserfahrungen des/r psychoanalytischen Psychotherapeuten/in unverzichtbar und die Entwicklung einer authentischen Haltung als Ausdruck seiner eigenen therapeutischen und religiösen Identität.
\end{abstract}

Schlüsselwörter: Interkulturelle Psychotherapie, Religiosität, Spiritualität, psychische Gesundheit, Glaubensgewissheit, Identitätswandel, Migration

\section{Einleitung}

Die psychiatrisch-psychotherapeutischen Heilmethoden werden von den kulturellen Weltbildern der Gesellschaften und Kontinente geprägt, in denen sie Anwendung finden. Die Religiosität und Spiritualität spielen in den kulturellen Weltbildern der Menschen je nach Säkularisierung einer Gesellschaft eine mehr oder weniger grosse Rolle. Welchen Beitrag zur Behandlung von Menschen mit psychischen Störungen Religion und Spiritualität leisten oder auch leisten können, ist eine Frage, mit der sich Psychotherapeut*innen in den weitgehend säkularisierten Gesellschaften Mitteleuropas in den vergangenen Jahrzehnten wenig zu beschäftigen brauchten. Psychiater*innen und Psychotherapeut"innen erwiesen sich als weniger religiös als ihre Patient*innen (Neeleman und Lewis, 1994). "Religion is psychiatry's last taboo", formulierte überspitzt Hans Küng diesen Tatbestand (katholischer Theologe, Universität Tübingen, 1986). Die Abwendung von Religion und Spiritualität hatte zur Folge, dass die Haltungen von Psychiater*innen und Psychotherapeut*innen bei der Beschäftigung mit den kulturellen Ausformungen von Religiosität und Spiritualität im interkulturellen Therapiesetting ein noch wenig der bewussten (Selbst-)Reflexion und Erforschung zugänglicher Erfahrungsbereich war. Dies hat sich in den vergangenen beiden Jahrzehnten gewandelt. Die gesellschaftliche Interkulturalisierung und die aktuell grössere Durchdringung des öffentlichen und privaten Raumes durch Religiosität und Spiritualität zum Beispiel durch den Islam, christliche, wie evangelikale und andere Glaubensrichtungen, hat nicht zuletzt auch in Mitteleuropa die religiöse Frage für die Psychiater*innen und Psychotherapeut*innen zu einer aktuellen aufgewertet. Welchen Sinn macht die Rückkehr der Religiosität und Spiritualität in die Psychiatrie und Psychotherapie? Bei möglichen Antworten muss es vorrangig um einen therapeutischen Wissens- und Methodenzugewinn und eine professionelle Haltung gehen, die immer auch in einer Interferenz mit den ganz persönlichen Glaubensüberzeugungen und der Identität der Psychiater*innen und Psychotherapeut*innen steht.

\section{Sinnstiftung als gemeinsame Aufgabe von Religiosität/Spiritualität und Psychotherapie?}

Aus den Schulen und Kulturen übergreifenden Erfolgsbilanzen unterschiedlicher traditioneller (z. B. spiritueller) und moderner therapeutischer Heilmethoden lässt sich sagen, dass eine Psychotherapie offenbar dann erfolgreich ist, wenn die Erklärungsmodelle und Behandlungsschritte des/r Therapeuten/in bzw. Heilers/in für das subjektive und kulturelle Weltverständnis des/r Patienten/in plausibel und nachvollziehbar sind. Nachvollziehbare sinnstiftende Konstruktionen zwischen Symptom und Diagnose sowie Ätiologie und Behandlungsmethode zeichnen sich kulturübergreifend durch ihre innere Schlüssigkeit aus und stellen ein wesentliches therapeutisches Element 
dar (Machleidt \& Passie, 2018, S. 521-531; Stöckigt \& Machleidt, 2018, S. 531-534). Die Glaubenseinstellungen der Patient*innen sind ein integrativer Teil seines Weltverständnisses. Der Beitrag der Religiosität und Spiritualität kann in dem Sinne eine sinnstiftende und sinnintegrierende Funktion haben, nämlich als ein handlungsübergreifendes und harmonisierendes Modell. Dies gilt ganz unabhängig von dem Akkulturationsniveau des Patienten und von der Tatsache, ob Dritte das benutzte Erklärungsmodell und die Behandlungsschritte im Sinne "wissenschaftlicher Erkenntnis» als richtig beurteilen oder auch nicht. An individuell geprägte Verständnismodelle lassen sich keine medizinisch- bzw. psychologisch-wissenschaftlichen Massstäbe anlegen. Sie sind an den subjektiven Erlebniswirklichkeiten und Verständnishorizonten der Patient*innen formulierte, harmonisierende Konstrukte (Machleidt \& Passie, 2018; Stöckigt \& Machleidt, 2018).

Das Unbehagen der westlichen Psychiater*innen und Psychotherapeut*innen, die Religiosität und Spiritualität des/r Patient*in für den Therapieerfolg zu nutzen, hat einen handfesten und berechtigten Grund. Dieser liegt in der tief verwurzelten Angst der Psychiater"innen und Psychotherapeut*innen vor dem Rückfall in bzw. der Abgrenzung gegenüber einem unaufgeklärten Dämonismus zum Beispiel in Gestalt religiöser Exorzismen oder ähnlicher Praktiken. Das Unbehagen rührt auch von der antizipierten «Unwissenschaftlichkeit» beim Rückgriff auf religiös-spirituelle Ressourcen her. Haben Religiosität und Spiritualität denn nachweisbare salutogenetische Wirkungen (siehe u.a. Kaiser, 2007; s. a. Machleidt, 2013)?

Aktuelle Forschungen zeigen, dass sich der Nutzen und die Grenzen des Einbezugs von Religiosität und Spiritualität in die westliche Psychiatrie und Psychotherapie ganz gut auch empirisch nachweisen lassen.

\section{Glaubensgewissheiten als therapeutische Ressource?}

Für eine Neufokussierung der Religiosität und Spiritualität ginge es zunächst einmal um die rationale Überzeugung auf dem Boden einer soliden empirischen Fundierung, dass religiöse und spirituelle Glaubensüberzeugungen und psychische Gesundheit positiv miteinander korrelieren. Metaanalysen zeigen, dass positive Emotionen, Wohlbefinden, Hoffnung und Zuversicht, positiver Lebenssinn und -zweck, Selbstachtung und -kontrolle einen positiven Bezug zu Glaubensüberzeugungen haben. Diese Fundierung ist wesentlich in den vergangenen beiden Jahrzehnten für depressive, Angst- und Somatisierungsstörungen sowie Sucht erarbeitet worden. Bei depressiven Störungen zum Beispiel sind Glaubensüberzeugungen verbunden mit einer schnellerer Remission (einer Verringerung ihres Schweregrads bei religiösen spirituellen Interventionen anhand von Fallbeobachtungen) sowie einer verringerten Prävalenz von Suizidgedanken und -handlungen. Entscheidend für die protektive Wirkung ist das Vorhandensein einer inneren Glaubensgewissheit und der damit verknüpften verinnerlichten Repräsentant*innen und Stifter*innen des Glaubens, wie bspw. in den drei abrahamitischen Religionen der Gott, im Christentum zudem der Erlöser, im Islam der Prophet etc. und die Kultivierung des Glaubens in der Gemeinschaft. Letzteres gilt übrigens religionsübergreifend für Gläubige aller drei abrahamitischen Religionen genauso wie auch für Hindus. Fragt man danach, wie sich Religiosität und Spiritualität auf die psychische Gesundheit konkret auswirkt, so geht es um eine erhöhte Resilienz. Eine höhere psychische Resilienz wird erreicht durch stabilisierende religiöse Einflüsse auf den Lebensstil, durch soziale Unterstützung, religiöse Stress- und Problembewältigung, kognitive Neubewertungen, Stress Reduktion, weniger Egozentrizität und durch transportierte religiöse und spirituelle Werte wie Vergebung, Altruismus und Dankbarkeit. An diesen plausiblen hilfreichen Kriterien ist bemerkenswert, dass sich darin seelsorgerische und therapeutische Anliegen überschneiden. In psychotherapeutischen Kontexten mit Gläubigen unterschiedlicher Religionszugehörigkeit kann es also durchaus Sinn machen, Glaubensgewissheiten kulturkompetent zu analysieren und als Ressource gezielt und differenziert in Therapieprozesse einfliessen zu lassen (Smith et al., 2003; Kaiser, 2007, S. 587-596; Blazer, 2012; Miller et al., 2012; Utsch et al., 2014, S. 111-115; Ohls \& Agorastos, 2018, S. 109ff.; für eine Übersicht siehe Dein, 2018).

Kommt es zum Verlust von Glaubensgewissheiten zum Beispiel bei Pfarrern*innen so resultieren Identitätsstörungen, Unsicherheiten in der professionellen Orientierung, Motivationseinbussen, Authentizitätsprobleme, Berufsabbruch und anderes im Zusammenhang mit depressiven Syndromen, Erschöpfungszuständen und Burn-out sowie Zukunftsängsten (Kasuistische Erfahrungen; Machleidt, 2018).

Irrational und befremdlich anmutende Glaubensüberzeugungen von Angehörigen zum Beispiel vorabrahamitischer Religionen wie dem Jesidentum ${ }^{1}$ oder hochreligiöser Migrant*innen der drei abrahamitischen oder der asiatischer Religionen erfordern über allen Respekt und differenzsensibles Einfühlungsvermögen hinaus ein interreligiöses Wissen. Dieses interreligiöse Wissen erleichtert den religionssensiblen Verständniszugang. Dieser muss aber durch die subtile Kenntnis und Analyse der subjektiven Glaubensgewissheiten des/r Patient* in vertieft werden, um individuelle therapeutische Relevanz zu bekommen. Die religiösen Glaubensinhalte und deren Ritualisierungen sind so etwas wie der Mantel, der sich dem inneren Kern der individuellen subjektiven Glaubensgewissheiten um die Schultern legt.

Beim professionellen Umgang mit fremden Glaubensüberzeugungen können vier Aspekte als wegweisend gelten, die den Psychiater*innen und Psychotherapeut*innen davor bewahren können, schnell an seine eigenen Grenzen zu kommen: eine Wertoffenheit und Bedachtsamkeit,

1 «Das Jesidentum ist eine monotheistische, nicht auf einer heiligen Schrift beruhende, synkretistische Religion. Die Mitgliedschaft ergibt sich ausschließlich durch Geburt, wenn beide Elternteile jesidischer Abstammung sind» (Wikipedia-Artikel). 
weltanschauliche Kompetenz, Wissen über die Bedeutung von Religiosität und eine Differenzierung zwischen weltanschaulichem und therapeutischem Angebot (Ohls \& Agorastos, 2018, S. 109). Kommt der/die Psychiater*in und Psychotherapeut*in trotz einer Berücksichtigung dieser vier Aspekte bei einem/r Gläubigen an seine/ihre eigenen ethischen Grenzen, so verlangt es das Authentizitätsgebot, diese in therapeutisch geeigneter Weise zu thematisieren mit dem Ziel, eine wechselseitige Akzeptanz von Verschiedenheit zu erarbeiten. Gelingt dies nicht, kann ein vorzeitiges einvernehmliches Therapieende wegen unauflösbarer negativer Übertragungen unvermeidlich sein. In jedem Falle sollte ein/e Supervisor*in eingeschaltet werden.

\section{Seelsorge und Psychotherapie im Dialog}

Seelsorge und Psychotherapie stehen in einem interessanten Dialogverhältnis zueinander, zu dem im Folgenden etwas aus der Sicht der Psychotherapie gesagt wird. Von den zahlreichen Versuchen Seelsorge in Abgrenzung zur Psychotherapie zu definieren, seien im Folgenden Formulierungen einiger Autor*innen/Seelsorger*innen beispielhaft genannt. Utsch zum Beispiel sagte (2002; zit. n. Kaiser, 2007, S. 596ff.):

«Die Aufgabe der Seelsorge besteht in der Freisetzung christlichen Verhaltens (Praxis) zur Lebensbewältigung. Im seelsorgerlichen Gespräch steht der von Gott geliebte und entfremdete Mensch mit seinen Konflikten im Mittelpunkt. Seelsorge geschieht in der Hoffnung, dass Gott dorthin kommt, wo sich Menschen auf den Weg zur Wahrheit machen.»

So definiert kann Seelsorge eine Praxis entwickeln, durch die Weiterleben gelernt werden kann. Bei der Methodik, mit der Seelsorge-Gespräche geführt werden, wird durchgängig der pastoral-psychologische Ansatz hervorgehoben, der auf psychotherapeutische Methoden Bezug nimmt, wie die Psychoanalyse, die systemische Therapie und insbesondere den personzentrierten Ansatz von Carl Rogers (s. Engemann, 2007). Ziel dessen ist es, Ratsuchenden das zu bieten, was in deren jeweils aktueller Situation hilfreich sein könnte. Das ist eine Haltung, die besonders für die Seelsorge von Bedeutung ist. Darüber hinaus ist die personzentrierte Haltung durch die Basisvariablen Echtheit, Akzeptanz und Empathie geprägt, die sich in den genuin christlichen Verhaltensweisen von Annahme, Wahrhaftigkeit und Liebe wiederfinden. Der personzentrierte Ansatz hält eine Distanz zu den zweckrationalen psychotherapeutischen Prozeduren wie systematische Anamneseerhebungen, Diagnostik, gezielte Therapieplanung und -methodik.

Die deutlichsten Unterschiede zwischen einer «absichtslos» unterstützenden Seelsorge und einer auf das Individuum fokussierten und auf Heilung zielenden Psychotherapie liegen im Rekurs der Seelsorge auf die spirituelle Dimension und in dem Aufbau und der Pflege der Gemeinschaftsbindung begründet.
Insbesondere Themen wie Trauer, Abschied, Verlust und Zukunftsängste sind originäre Themen der Psychotherapie und der Seelsorge auch bei Menschen aus anderen Kulturen. Wie begegnen aber Vertreter"innen unterschiedlicher Weltreligionen ihren Gläubigen aus anderen Ländern und Kulturen? Grosse Bedeutung haben dabei gemeinsame religiöse Praktiken, die in allen Weltreligionen zu finden sind, wie das Sprechen von Gebeten, das Lesen heiliger Schriften und das Anhören von Textauslegungen (Predigten). Diese Praktiken sind häufig verbunden mit einem liturgischen Ritual unter Leitung eines Geistlichen. Solche Rituale dienen der Trauerbewältigung, der Entängstigung und dem Wecken von Zuversicht und positiven Erwartungen. Schuld und Sühne durch Vergebung und Trost sind verbreitete spirituelle Interventionen, die Weiterleben möglich machen und Zukunft eröffnen sollen. Sie werden in der Gemeinschaft der Gläubigen im Rahmen liturgischer Rituale wie zum Beispiel dem Abendmahl praktiziert.

Mit dem Glauben wird eine "spirituelle Wirklichkeit» ins Spiel gebracht, «die höher ist als alle Vernunft» und grösser ist als die des Trauernden. Das eigene Werden und Vergehen in der Hand eines Höchsten zu sehen, ist ein machtvolles spirituelles Prinzip, das Tröstung, Versöhnung und Heilung durch religiöse Sinnstiftung ermöglicht. Man kann dieses Prinzip wissenschaftlich den «spirituellen Heilfaktor» nennen, dessen stärkenden Wirkungen den Gläubigen in den Weltreligionen teilhaftig werden und dessen ethnopsychologische Frühformen in der traditionellen Heilkunst indigener Gesellschaften seit Jahrhunderten in einer grossen Vielfalt mit Erfolg praktiziert wurden (Ohls \& Agorastos, 2018, S. 109; Machleidt \& Passie 2018; Stöckigt \& Machleidt 2018).

\section{Religion als Diskursvielfalt}

Was ist Religion und welche subjektiven Überzeugungen lassen sich unter den Begriff der «Religion» subsummieren? Religion ist ja einer der Leitbegriffe mit denen wir hier diskutieren. Wenn wir Religionsexpert*innen fragen, dann erfahren wir zum Beispiel das Folgende: "In der wissenschaftlichen Religionsforschung ist die Unterscheidung zwischen dem, was man «Religion` nennen möchte und dem, was man nicht so nennen möchte, derart kontrovers, dass nicht einmal Einigkeit über einen 〈Kernbereich des Religiösen` besteht» (Popp-Baier, 2007, S. $515 \mathrm{ff}$. .). Den diskursiven Charakter dieses Begriffs und die Spielräume, die er lässt, nehmen psychologische Psychotherapeut*innen gern zur Kenntnis. Den therapeutischen Anliegen kommen Antworten entgegen, wie die, dass das Wort Religion keinen identifizierbaren Gegenstandsbereich benennt, also eine ganze Vielfalt unter diesen Begriff subsummiert wird. Zum anderen benennt dieses vieldeutige Wort vorrangig «Diskurse», die viele unterschiedliche Debatten einbeziehen wie wissenschaftliche, öffentlich-gesellschaftliche und private. Es ginge dann darum, den Blick für eine Pluralität dieser Diskurse und ihre Diversität und Heterogenität zu öffnen, und 
damit eine Unterdrückung oder Ausgrenzung bestimmter Gruppierungen und Individuen zu vermeiden. Der realen Glaubensvielfalt in den Diskursen der Menschen, denen wir in unseren Behandlungen begegnen, steht die Behauptung einer «Kernsubstanz» oder eines «unveränderlichen Glaubenskerns» gegenüber, die meistens von einer Glaubenselite formuliert und verteidigt werden. Im Gegensatz dazu praktizieren der/die Einzelne und verschiedene Gruppierungen «ihre Religion» vor Ort im Kontext ihrer Lebenssituation und handeln ihr Religionsverständnis diskursiv aus. Der/die einfache Gläubige weiss meist nur wenig von den Diskursen «der da oben», der Glaubenseliten oder wie es in Indien heisst: Der «Dalit» (der Unberührbare, niedrigste Kaste) hat meist nur wenig vom Diskurs der «Brahmanen» (höchste Kaste) (ebd., S. 522f.).

Die Vielfalt der Diskurse und Praktiken hat eine Vielfalt der Glaubensrichtungen und eine Breite theologischer Deutungsmöglichkeiten hervorgebracht, für die gerade der Islam mit seiner über Jahrhunderte liberalen und differenzierten Diskurskultur ein hervorragendes Beispiel abgegeben hat. Die aktuellen dogmatischen Engführungen der islamischen Lehre beruhen auf der Dominanz der sunnitischen Glaubensrichtung und weichen drastisch von den liberalen Traditionen des Islams ab, die einen gleichmachenden Universalismus, der kulturelle Differenzen unterschlägt, nicht zugelassen haben. Die bestimmenden Werte des Islam unterscheiden sich in ihrer Substanz nur wenig von denen der beiden anderen abrahimitischen und auch von denen anderer grosser Religionen (Seidel, 2018, S. 190). Das heisst nicht, dass nicht schon immer auch fundamentalistische Bewegungen in den Weltreligionen ihre Stimme in die religiösen Diskurse eingebracht haben wie innerhalb des Christentums, Judentums, Islam, Buddhismus, Hinduismus etc. Bei diesen fundamentalistischen Strömungen wurden mit Bezug auf heilige Schriften «bestimmte Dogmen und Praktiken ausgewählt, die keinem Widerspruch mehr zugänglich sind und die Lebensführung des Einzelnen und ganzer Gesellschaften» zu bestimmen suchen (siehe zuvor). Diese stehen heute vielfach im Brennpunkt von Politik und Öffentlichkeit, stellen aber gleichwohl ein Minderheitenphänomen dar im Kontext der vielen Glaubensdiskurse, die unter den Begriff «Religion» subsummiert werden können.

\section{Fremdheitserfahrungen: «Die Fremden rücken immer näher!»}

Im Spannungsfeld zwischen religiöser Diskursvielfalt einerseits und zunehmender Dogmatisierung andererseits sind Migrationsprozesse von Menschen mit unterschiedlichsten Glaubensorientierungen verortet. Migrationsprozesse geben wichtige Impulse für Veränderungen religiöser Glaubensüberzeugungen als identitätsbildende Strukturen der Betroffenen und konfrontieren die Einheimischen mit fremd anmutenden Orientierungen sowohl im öffentlichen wie im privaten Raum. Wie gut die damit einhergehenden wechselseitigen Fremdheitserfahrungen bewältigt werden können, ist entscheidend für die Qualität der interkulturellen Beziehungen in den Aufnahmegesellschaften. In unseren psychotherapeutischen Arbeitsbeziehungen sind wir wegen der entstehenden Nähe und als Mitwissende intimer subjektiver Glaubensüberzeugungen darum bemüht, die Fremdheitserfahrungen, die wir dabei machen, ausreichend gut zu bewältigen. Denn in interkulturellen Therapieverläufen sind wir häufig zu expliziten Antworten aufgefordert oder finden uns in bewusst/unbewussten Reaktionsmustern wieder. Antworten erfordern ein explizites vorurteilsfreies Wissen über die Glaubensgewissheiten und deren Bedeutungen für unsere Patient*innen. Aversive Reaktionsmuster unsererseits machen es erforderlich, sich selbst über die Schulter zu sehen oder in Supervisionen sich über die Schulter sehen zu lassen, um nicht eigene dysfunktionale oder destruktive Impulse unbemerkt in die interkulturellen bzw. interreligiösen Therapieprozesse einfliessen zu lassen.

Die Bewältigung von Fremdheitserfahrungen ist ein wesentliches Ich-konstituierendes Merkmal individueller professioneller Entwicklung von psychologischen Psychotherapeut*innen in globalen kulturellen Kontexten, weil die «Fremden immer näher rücken». Die damit aufkommenden Ängste haben zu dem verbreiteten Irrtum beigetragen, dass durch die Fremden die eigene Identität und Ich-Entwicklung blockiert würden. Vielmehr verhält es sich umgekehrt. Ohne das Fremde gäbe es kein Eigenes. Schliesslich ist das Fremde nicht zuletzt in seiner religiösen Gestaltung eine ständige von Neugier und Angst begleitete Herausforderung, die eigenen begrenzten Näheräume zu verlassen und sich auf den Weg zu neuen Erfahrungshorizonten aufzumachen (Machleidt et al., 2018). Wenn ich Religionen trotz aller Unbestimmtheit ihres Gegenstandes als plurale Symbolsysteme verstehe, die eine Weltordnung mit einem Lebensstil verbinden oder als «Wegbeschreibungen», die dem Einzelnen «seinen Lebensweg verdeutlichen, gegenwärtigen Erfahrungen Sinn verleihen und Anweisungen für künftiges Handeln geben können» (Popp-Baier, 2007, S. 518f.), dann sind dies brauchbare entängstigende Definitionen, die universell verstanden werden können und therapeutischen Anliegen nahe stehen. In ihren so definierten Kernanliegen rücken Religionen in eine therapeutische Nähe, auch wenn sie in ihren unterschiedlichen Dogmen und Ritualen in Kirchen, Moscheen, Synagogen oder Tempeln Fremdheitsgefühle vermitteln können.

Psychologische Psychotherapeut*innen könnten sich eine Reihe von Fragen zu ihren individuellen Reaktionen und ihren ganz eigenen Versuchen stellen, religionsaffine Fremdheitsgefühle zu bewältigen.

$>$ Geht es mir darum, Abstand zu religiösen Menschen zuhalten, ihre Religion auszublenden, zu ignorieren, die Thematisierung zu vermeiden, ggf. die Flucht vor solchen Themen zu ergreifen?

> Oder erlebe ich Neugier auf das Religionsverständnis der Anderen, habe ich Interesse und verbinde hilfreiche Einsichten für die Behandlung damit? Idealisiere ich einen Anderen, weil er einer bestimmten Religion an- 
gehört, zum Beispiel dem Buddhismus, verbinde ich Heilserwartungen mit einer bestimmten Religion, suche einen Guru oder versuche ich Abstand zu halten?

> Verstehe ich mein Fremdheitsgefühl zum Anderen als ein Kennzeichen meiner Beziehung zu ihm? Mache ich Versuche, Gemeinsamkeiten zu finden und Konsense herzustellen, und führt das zu einem für mich erwünschten Beziehungswandel im Sinne grösserer Vertrautheit?

$>$ Oder versuche ich Fremdheit zum Verschwinden zu bringen? Habe ich die (narzisstische) Fantasie, alle Menschen könnten oder sollten eigentlich so sein oder so werden wie wir, ein «Ab(zieh)bild» unserer selbst? Stören die Fremden deshalb und sollten neutralisiert werden oder aus dem öffentlichen Raum und meinem Sprechzimmer verschwinden?

> Erwarte ich die Neutralisierung des Fremden durch Akkulturation und Integration bzw. durch die Vereinnahmung durch uns? Habe ich den Drang, alles Fremde zu meiner eigenen Harmonisierung vollständig verstehen zu müssen, oder kann ich dem Respekt vor seiner situativen Unzugänglichkeit Raum geben?

$>$ Kann ich mich dem spannungsreichen Wechselspiel zwischen meinen Erwartungen an die Fremden und dem realen Bild der Alltagserfahrung mit den Fremden überlassen? Gelingt es mir, Distanzierung und Wiederannäherung wie in einer Pendelbewegung immer wieder neu auszubalancieren im Sinne einer «Optimaldistanz»?

Soweit einige Fragen an uns zur Selbstvergewisserung.

\section{Therapeutischer Identitätswandel?}

Psychiater"innen und Psychotherapeut"innen sollten sich der Frage öffnen, welche Haltung sie selbst authentisch gegenüber Menschen mit ausgeprägten subjektiven Glaubensgewissheiten vertreten können als Ausdruck ihrer eigenen therapeutischen Identität. Verinnerlicht haben viele von uns die vielfach auch bestrittene These des Sozialwissenschaftlers und Religionssoziologen Max Weber (1988 [1920]), die besagt, dass als Folge des fortschreitenden Modernisierungsprozesses das religiöse Element in der Gesellschaft abnehme bzw. ganz verschwinde und dass Religion privatisiert und marginalisiert werde (s.a. die EKD-Studie 2018 zur «postchristlichen Generation» der 20-30-jährigen). Je nachdem, wie ich zu dieser Säkularisierungstheorie stehe, betrachte ich religiöse Menschen oder Migrantengruppen ggf. als rückständig, regressiv, «kindlich»-abhängig, magischem (Schuld- und Sühne-)Denken verhaftet etc. Mit solchen Konnotationen antizipiere ich ein Kultur- bzw. Modernitätsgefälle zwischen mir selbst als Vertreter*in einer «säkularisierten mitteleuropäischen Hochkultur» und den fremden Gläubigen, das an kolonialistische Einstellungen erinnert. So eine asymmetrische Begegnungssituation kann nicht förderlich für die Knüpfung hilfreicher interkultureller Arbeitsbeziehungen in psychotherapeutischen Settings sein. Therapeut*innen müssen sich kritische Rechenschaft ablegen über ihre Haltung zu religiösen Menschen, da Beziehungsknüpfungen in therapeutischen Prozessen ohne Wertschätzung auf Augenhöhe und Authentizität als Orientierung vermittelndes Element nicht gelingen können. Die Identität von Menschen lässt sich ja nicht auf eine Dimension ihrer Persönlichkeit reduzieren wie zum Beispiel das Frausein, das Türkischsein oder das Moslemsein etwa. Solche Zuschreibungen einer partiellen Identität laufen Gefahr, Menschen, die ein so verengter Blick nicht wahrzunehmen vermag, aus ihren Betrachtungen auszuschliessen (Seidel, 2018, S. 190).

Natürlich dürfen und müssen auch persönliche Grenzen therapeutischer Toleranz in Betracht gezogen, diskutiert und respektiert werden. Es existiert ja nicht so etwas Allgemeinverbindliches wie eine «Meta(welt)kultur», die überall und bei jedem Akzeptanz findet. Vielmehr existieren multipolare kulturelle Bezugssysteme in denen mit unterschiedlichsten Glaubensidentitäten Daseinsorientierung gelebt werden kann. In einer solchen religionspluralen Weltgesellschaft entstehen im Kontext von Migration Sorgen und Ängste um den Erhalt der eigenen religiösen Identität auch im christlich säkular geprägten Mitteleuropa und eine Eskalation von unheilvollen Kulturkämpfen wie im Nahen Osten, im Kaukasus etc. (Essen, 2007, S. 291f.). Dass ich als Therapeut zu diesem Zeitgeschehen innerlich Stellung beziehe und Grenzen meiner Toleranz definiere, ist nachvollziehbar. Dazu ein Beispiel: Es gibt besorgniserregende Unterschiede bei verschiedenen religiösen Migrant*innengruppen hinsichtlich ihrer Integrationsbereitschaft. In der vietnamesischen Diaspora zum Beispiel wird in den transnationalen Kommunikationsräumen die Ahnenverehrung weiter integrationsfördernd kultiviert genauso wie in den unter Westafrikaner"innen populären Pfingstkirchen. Dagegen liefert die islamistische Salafi-Bewegung ein Beispiel für Abgrenzung und Verwerfung integrationsfördernder pluralistischer sozialer und religiöser Lebensstile. Wenn Psychiater"innen und Psychotherapeut"innen diese Unterschiede wahrnehmen, vermögen sie ihre eigenen therapeutischen Haltungen, ihre Toleranzgrenzen wie ihre Spielräume genauer zu bestimmen (Bazu \& Gies-Powroznik, 2018, S. 20f.).

Eine günstige Voraussetzung für interreligiöse Erkundungen sind psychologische Psychotherapeut*innen, die Neugier und Interesse daran entwickeln, welche "Götter und Geister» sie selbst und die Seelenlandschaften ihrer interkulturellen Patient"innen bevölkern. Dieses Interesse bietet die Chance, die eigene Wahrnehmung dafür zu schärfen, wie diese beschaffen sind und mit welchen Eigenschaften und Funktionen sie ausgestattet sind. Menschen mit inneren Glaubensgewissheiten haben sich ein kulturell vorgeprägtes, aber bei genauerem Hinsehen individuelles inneres positives Objekt konstruiert. Dieses Sicherheit gebende Objekt ist mit prägnanten kulturell vermittelten Eigenschaften und mit je spezifischen Funktionen für die Bewältigung unterschiedlichster Lebenssituationen ausgestattet. Häufig repräsentieren diese Zuschreibungen auf das positive innere Objekt wie in einem Spiegelbild die Eigenschaften dieser Menschen selbst im idealisierten 
positiven wie auch negativen Sinne, als so etwas wie die eigenen Stärken und Schwächen. Wie mit einem Vergrösserungsglas lassen sich diese, als den transzendenten Figuren ihres Glaubens auf den Leib geschneidert, betrachten. Ein vertieftes Verständnis des eigenen wie des fremden Selbst lässt sich aus diesem Spiegelbild gewinnen und die zum Beispiel in einer Behandlung zur Disposition stehende Konfliktdynamik differenzierter analysieren und bearbeiten.

\section{Selbstidentität im Wandel}

Der Wandel der Selbstidentität von Psychiater*innen und Psychotherapeut*innen bei der Begegnung mit dem religiösen und kulturellen Fremden ist ein universelles individuelles und gesellschaftliches Phänomen angesichts der globalen Wanderungsbewegungen durch transnationale Migration. Der postmodernen Selbstidentität von Psychiater*innen und Psychotherapeut*innen eröffnet dieser Wandel die Chance, sich in einer grösseren kulturellen Fliessfähigkeit zu üben, als einem inneren Mobilitätszugewinn, der auch die erhöhte äussere Mobilität und Vielfalt unserer Lebenswelt widerspiegelt. Die kontextuelle und geografische Mobilität und Vielfalt als dynamische Charakteristik postmoderner Lebenswege lässt sich als einen individuellen, professionellen und zivilisatorischen Zugewinn verstehen, der allerdings nicht ohne massive innere und äussere Widerstände und Krisen zu erringen ist.

\section{Literatur}

Bazu, H. \& Gies-Powroznik, N. (2018). Ethnologische Aspekte der Migration. In W. Machleidt, U. Kluge, M. Sieberer \& A. Heinz (Hrsg.), Praxis der interkulturellen Psychiatrie und Psychotherapie. Migration und psychische Gesundheit (S. 15-22). 2. Aufl. München: Elsevier Urban \& Fischer.

Blazer, D. (2012). Religion/Spirituality and Depression: What Can We Learn From Empirical Studies? Am J Psychiatry, 169(1), 10-12.

Dein, S. (2018). Culture, Religion and Mental Health. Lecture. 5th World Congress of Cultural Psychiatry, Oct. 10th, New York.

Engemann, W. (Hrsg.). (2007). Handbuch der Seelsorge. Grundlagen und Profile. Leipzig: Evangelische Verlagsanstalt.

Essen, G. (2007). Interkulturelle Theologie. In J. Straub, A. Weidemann \& D. Weidemann (Hrsg.), Handbuch der interkulturellen Kommunikation und Kompetenz (S. 283-293). Stuttgart und Weimar: J. B. Metzler.

Kaiser, P. (2007). Religion in der Psychiatrie. Eine (un)bewusste Verdrängung? Göttingen: V\&R unipress.

Küng, H. (1986). Religion: The Last Taboo. Washington, D.C.: APA Press.

Machleidt, W. (2013). Migration, Kultur und psychische Gesundheit. Dem Fremden begegnen. Stuttgart: Kohlhammer.

Machleidt, W. (2018). unveröffentlichtes Manuskript.

Machleidt, W., Kluge, U., Sieberer, M. \& Heinz, A. (Hrsg.). (2018). Praxis der interkulturellen Psychiatrie und Psychotherapie. Migration und psychische Gesundheit. 2. Aufl. München: Elsevier Urban \& Fischer.

Machleidt, W. \& Passie, T. (2018). Traditionelle Heilkunst und moderner Interaktionismus im Dialog. In W. Machleidt, U. Kluge, M. Sieberer \& A. Heinz (Hrsg.), Praxis der interkulturellen Psychiatrie und Psychotherapie. Migration und psychische Gesundheit (S. 521-531). 2. Aufl. München: Elsevier Urban \& Fischer.
Miller, L., Wickramaratne, P., Gameroff, M.J., Sage, M., Tenke, C.E. \& Weissmann, M.M. (2012). Religiosity and Major Depression in Adults at High Risk: A Ten-Year Prospective Study. Am J Psychiatry, 169(1), 89-94.

Neeleman, J. \& Lewis, G. (1994). Religious identity and comfort beliefs in three groups of psychiatric patients and a group of medical controls. International Journal of Social Psychiatry, 40, 124-134.

Ohls, I. \& Agorastos, A. (2018). Religion und Migration. In W. Machleidt, U. Kluge, M. Sieberer \& A. Heinz (Hrsg.), Praxis der interkulturellen Psychiatrie und Psychotherapie. Migration und psychische Gesundheit (S. 103-111). 2. Aufl. München: Elsevier Urban \& Fischer.

Popp-Baier, U. (2007). Religion. In J. Straub, A. Weidemann \& D. Weidemann (Hrsg.), Handbuch der interkulturellen Kommunikation und Kompetenz (S. 515-524). Stuttgart und Weimar: J.B. Metzler.

Seidel, R. (2018). Interkulturelle Kompetenz. In W. Machleidt, U. Kluge, M. Sieberer \& A. Heinz (Hrsg.), Praxis der interkulturellen Psychiatrie und Psychotherapie. Migration und psychische Gesundheit (S. 189-197). 2. Aufl. München: Elsevier Urban \& Fischer.

Smith, T. B., McCullough, M.E. \& Poll, J. (2003). Religiousness and depression: evidence for a main effect and the moderating influence of stressful life events. Psychol Bull, 129, 614-636.

Stöckigt, B. \& Machleidt, W. (2018). Spirituelle Heilrituale in Ostafrika. In W. Machleidt, U. Kluge, M. Sieberer \& A. Heinz (Hrsg.), Praxis der interkulturellen Psychiatrie und Psychotherapie. Migration und psychische Gesundheit (S. 531-534). 2. Aufl. München: Elsevier Urban \& Fischer.

Utsch, M., Bonelli, R.M. \& Pfeiffer, S. (2014). Psychotherapie und Spiritualität. Springer: Heidelberg.

Weber, M. (1988 [1920]). Die Wirtschaftsethik der Weltreligionen. Vergleichende religionssoziologische Versuche. In M. Weber (Hrsg.), Gesammelte Werke zur Religionssoziologie (S. 273-573). Tübingen: UTB.

Wikipedia-Artikel: «Jesiden». https://de. wikipedia.org/wiki/Jesiden (13.01.2019).

\section{Religiousness and Spirituality in Intercultural Psychotherapy. Effects, methods and the identity of the therapist}

Abstract: The social interculturalisation and the greater influence of Islam in Central Europe, including religious beliefs in the public and private spheres, have given the religious orientations of psychotherapists and their intercultural patients greater topicality. Empirical studies have shown that pronounced religious convictions for depressive, anxiety and somatisation disorders as well as addiction correlate with increased psychological resilience across religions. Religions can be understood as plural symbolic systems that combine a world order with a lifestyle. The subtle knowledge of the beliefs allows a look at the inner psychic processes and opens wide and individual scope for therapeutic interventions involving religiosity and spirituality. The psychological psychotherapist's coping with the interreligious experiences of foreignness is indispensable for the positive connection to the patient and the development of an authentic attitude as an expression of his own therapeutic and religious identity.

Key Words: Intercultural Psychotherapy, Religiousness, Spirituality, Mental Health, Faith Certainty, Identity Change, Migration 
Religiosità e spiritualità nella psicoterapia interculturale. Effetti, metodi e identità del terapeuta

Riassunto: L'interculturalità sociale e la maggiore influenza dell'Islam e di altre fedi nella sfera pubblica e privata in Europa centrale hanno dato maggiore attualità agli orientamenti religiosi degli psicoterapeuti* e dei loro pazienti interculturali*. Studi empirici hanno dimostrato che le convinzioni religiose pronunciate per i disturbi depressivi, d'ansia e di somatizzazione, così come la dipendenza, sono correlate ad una maggiore resilienza psicologica tra le religioni. Le religioni possono essere intese come sistemi simbolici plurali che combinano un ordine mondiale con uno stile di vita. La sottile conoscenza delle credenze permette di guardare ai processi psichici interiori ed apre ampie e individuali possibilità di interventi terapeutici che coinvolgono la religiosità e la spiritualità. La capacità dello psicoterapeuta psicoanalitico di affrontare le esperienze interreligiose di estraneità è indispensabile per il legame positivo con il paziente e lo sviluppo di un'autentica attitudine come espressione della propria identità terapeutica e religiosa.

Parole chiave: psicoterapia interculturale, religiosità, spiritualità, salute psichica, certezza di fede, cambiamento di identità, migrazione

\section{Der Autor}

Wielant Machleidt, Prof. em. Dr. med., (Sozial-)Psychiater, Psychotherapeut und Psychoanalytiker (DPG), war von 1994 bis 2007 Inhaber des Lehrstuhls für Sozialpsychiatrie und Direktor der Abteilung Sozialpsychiatrie und Psychotherapie an der Medizinischen Hochschule Hannover. Seine Arbeitsschwerpunkte in Forschung und Lehre liegen auf dem Gebiet der interkulturellen Psychiatrie und der kultursensiblen Psychotherapie. Er war Vorsitzender des Referats für Transkulturelle Psychiatrie und Migration der Deutschen Gesellschaft für Psychiatrie, Psychotherapie und Nervenheilkunde (DGPPN) von 1994-2010, er ist Leiter der Supervisionsgruppe für interkulturelle Psychiatrie und Psychotherapie der ÄKN in Hannover und Ehrenvorsitzender des Ethno-Medizinischen Zentrums Hannover (EMZ). Zahlreiche wissenschaftliche Publikationen sind in Fachzeitschriften und als Buchveröffentlichungen verfügbar.

\section{Kontakt}

Prof. Dr. med. Wielant Machleidt

Kaiser-Wilhelm-Str. 18

30559 Hannover

E-Mail: wielant.machleidt@t-online.de 